\title{
Effect of Ester Compounds on Stereospecificity in Methacrylate Polymerization with $t$-Butyllithium/Bis(2,6-di-t-butylphenoxy)methylaluminum
}

\author{
Tomohiro Hirano, Tatsuki Kitayama, and Koichi Hatada
}

\author{
Department of Chemistry, Graduate School of Engineering Science, Osaka University, \\ Toyonaka, Osaka 560-8531, Japan
}

(Received April 13, 1998)

\begin{abstract}
Polymerization of methacrylates with $t$-butyllithium/bis(2,6-di- $t$-butylphenoxy)methylaluminum in toluene at low temperature gives heterotactic polymers which comprise an alternating sequence of meso $(m)$ and racemo $(r)$ diads. In this paper, the effect of addition of non-polymerizable esters on the stereoselectivity in heterotactic polymerization was examined. In the polymerization of methyl methacrylate, the addition of either bulky or less bulky esters scarcely affects the stereoselectivity of propagating anions. In contrast, the addition of bulky esters such as ethyl pivalate in the polymerization of ethyl methacrylate (EMA) increased heterotactic-selectivity of $m$-ended anions slightly without sacrificing high heterotactic-selectivity of $r$-ended anions, while the addition of less bulky esters such as ethyl isobutyrate decreased heterotactic-selectivity of $r$-ended anions drastically with a slight increase in heterotactic-selectivity of $m$-ended anions. The use of ethyl pivalate as a component of mixed solvent with toluene at $-95^{\circ} \mathrm{C}$ improved stereoselectivity of both $m$-ended and $r$-ended anions, giving heterotactic poly(EMA) with $m r=94 \%$.
\end{abstract}

KEY WORDS Heterotactic Polymer / Polymethacrylate / Stereoregularity / Stereospecific Polymerization / Living Polymerization /

Internal and external aromatic esters, such as ethyl benzoate or methyl $p$-toluate, are known to increase the stereospecificity in olefin polymerization with heterogeneous Ziegler-Natta catalysts such as $\mathrm{MgCl}_{2}$-supported Ti catalysts. The internal electron donors suppress the formation of non-stereospecific sites, ${ }^{1}$ and the external electron donors convert the non-stereospecific sites to the high isotactic-specific ones, and the low isotacticspecific sites to inactive ones. ${ }^{2,3}$

Ester compounds, as weak Lewis bases, have also been used in ionic polymerization systems as the third initiator component. Cationic polymerization of isobutyl vinyl ether (IBVE) with IBVE-acetic acid adduct/EtAlCl ${ }_{2}$ in the presence of ester additive, such as ethyl acetate and ethyl benzoate, proceeds in a living manner, while in the absence of the Lewis base neither living nor long-lived propagating species are formed under the same conditions. ${ }^{4,5}$

Anionic polymerization of methacrylates with $t$-butyllithium $(t-\mathrm{BuLi}) /$ trialkylaluminum in toluene at low temperatures gives syndiotactic polymers with narrow molecular weight distribution (MWD). ${ }^{6}$ However, Müller et al. found that this polymerization of methyl methacrylate (MMA) at $-78^{\circ} \mathrm{C}$ deviated significantly from conventional first-order kinetics due to the formation of a coordinative polymer network as gel in which the living chain ends are coordinated with carbonyl groups in the chain. ${ }^{7}$ They claim that the addition of ester compounds such as methyl pivalate and methyl benzoate prevents the formation of the coordinative network of living polymer chains, giving first-order time-conversion plots in the temperature range of -78 to $0^{\circ} \mathrm{C} .{ }^{7,8}$

We have reported that polymerization of methacrylates with $t$-BuLi/bis(2,6-di- $t$-butylphenoxy)methylaluminum $\left[\mathrm{MeAl}(\mathrm{ODBP})_{2}\right]$ in toluene at low temperature proceeded in a living manner and gave heterotactic polymers that comprise alternating sequence of meso $(m)$ diad and racemo $(r)$ diad (eq 1).9,10 The formation of

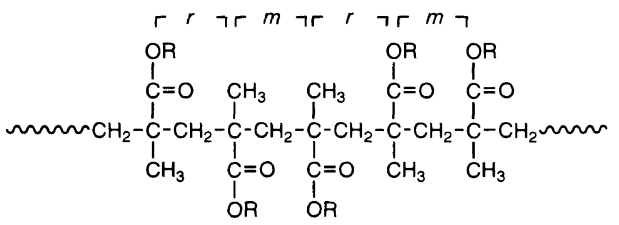

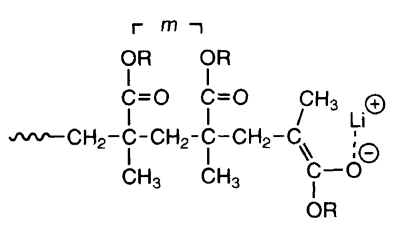

m-ended anion

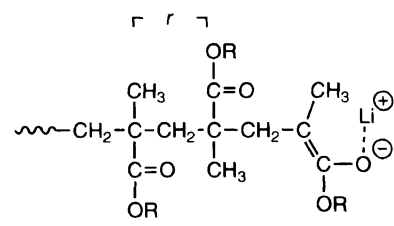

r-ended anion heterotactic polymer requires alternating stereoselections of the propagating anions, that is, $r$-addition by $m$-ended anions and $m$-addition by $r$-ended anions (eq 2 ). It is obviously higher order stereocontrol than those for isotactic and syndiotactic polymers. Stereochemical analysis of heterotactic poly(ethyl methacrylate) [poly(EMA)] by ${ }^{13} \mathrm{C}$ NMR revealed that heterotactic-selectivity ( $m$-selectivity) of $r$-ended anions was higher than heterotactic-selectivity ( $r$-selectivity) of $m$-ended anions. The chain-end analysis showed that (1) dimer anion favored $r$-addition, (2) the resultant $r$-trimer anion favored $m$-addition, and (3) $r$-ended anion was more abundant than $m$-ended anion in this polymerization system. ${ }^{11}$ Moreover, the NMR analysis at the terminating chain-end revealed that stereoregularity near the terminating chain-end decreased with increasing polymer yield. $^{12}$ This means that stereoselectivity of the propagating anions is affected by the monomer concentration. Since the monomer, ethyl methacrylate (EMA), is also an ester compound, the results imply the possibility that certain ester compounds may affect the stereospecificity of this polymerization. In this work, we examined the effect of non-polymerizable ester compounds (as the third initiator component) on the stereoselectivities of the propagating anions with the aim of 
Table I. Polymerization of MMA with $t$-BuLi/MeAl(ODBP $)_{2} /$ ester compound in toluene at $-78^{\circ} \mathrm{C}$ for $24 \mathrm{~h}^{\mathrm{a}}$

\begin{tabular}{|c|c|c|c|c|c|c|c|c|}
\hline \multirow{2}{*}{ Ester } & \multirow{2}{*}{$\frac{\text { Yield }}{\%}$} & \multirow{2}{*}{$\bar{M}_{n}^{\mathrm{b}}$} & \multirow{2}{*}{$\frac{\bar{M}_{w}{ }^{b}}{\bar{M}_{n}}$} & \multicolumn{3}{|c|}{ Tacticity $/ \%^{\mathrm{c}}$} & \multirow{2}{*}{$P_{m / r}^{\mathrm{d}}$} & \multirow{2}{*}{$P_{r / m}^{\mathrm{d}}$} \\
\hline & & & & $m m$ & $m r$ & $r r$ & & \\
\hline None & 100 & 8330 & 1.18 & $11.6^{\mathrm{e}}$ & $67.8^{\mathrm{e}}$ & $20.6^{\mathrm{e}}$ & 0.745 & 0.622 \\
\hline $\mathrm{CH}_{3}-\mathrm{C}_{0}-\mathrm{OC}_{2} \mathrm{H}_{5}$ & 44 & 4290 & 1.14 & 11.5 & 64.0 & 24.5 & 0.736 & 0.566 \\
\hline$\underset{\mathrm{CH}}{\stackrel{\mathrm{C}}{\mathrm{C}} \mathrm{H}_{3}} \underset{\mathrm{O}}{\stackrel{\mathrm{C}}{\mathrm{C}}-\mathrm{OC}_{2} \mathrm{H}_{5}}$ & 80 & 6190 & 1.26 & 11.4 & 67.2 & 21.4 & 0.747 & 0.611 \\
\hline 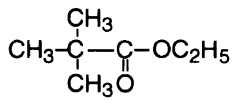 & 99 & 8620 & 1.38 & 12.2 & 69.2 & 18.6 & 0.739 & 0.650 \\
\hline & 99 & 8010 & 1.29 & 14.2 & 68.9 & 16.9 & 0.708 & 0.671 \\
\hline $\mathrm{II}-\mathrm{OCH}_{3}$ & 92 & 7010 & 1.24 & 11.8 & 66.7 & 21.5 & 0.739 & 0.608 \\
\hline $\mathrm{CH}_{3}-\stackrel{\mathrm{CH}}{\mathrm{C}}_{\mathrm{CH}_{3} \mathrm{O}}^{\mathrm{C}}-\mathrm{OCH}_{3}$ & 99 & 6420 & 1.31 & $11.2^{\mathrm{e}}$ & $67.3^{\mathrm{e}}$ & $21.5^{\mathrm{e}}$ & 0.750 & 0.610 \\
\hline $\mathrm{OCH}_{3}$ & 100 & 8170 & 1.12 & $11.5^{\mathrm{e}}$ & $66.9^{e}$ & $21.6^{\mathrm{e}}$ & 0.744 & 0.608 \\
\hline
\end{tabular}

a $t$-BuLi $0.2 \mathrm{mmol}$, MeAl(ODBP) $21.0 \mathrm{mmol}$, ester compound $1.0 \mathrm{mmol}$, MMA $10 \mathrm{mmol}$, toluene $10 \mathrm{ml}$. Ester compounds were added at $10 \mathrm{~min}$ after initiation. ${ }^{\mathrm{b}}$ Determined by GPC. ${ }^{\mathrm{c}}$ Determined by ${ }^{1} \mathrm{H}$ NMR. ${ }^{\mathrm{d}}$ The conditional probabilities in the first-order Markovian statistics. ${ }^{\mathrm{e}}$ Determined by ${ }^{13} \mathrm{C}$ NMR.

Table II. Polymerization of EMA with $t$ - $\mathrm{BuLi} / \mathrm{MeAl}(\mathrm{ODBP})_{2} /$ ester compound in toluene at $-78^{\circ} \mathrm{C}$ for $24 \mathrm{~h}^{\mathrm{a}}$

\begin{tabular}{|c|c|c|c|c|c|c|c|c|}
\hline \multirow{2}{*}{ Ester } & \multirow{2}{*}{$\frac{\text { Yield }}{\%}$} & \multirow{2}{*}{$\bar{M}_{n}^{\mathrm{b}}$} & \multirow{2}{*}{$\frac{\bar{M}_{w}^{b}}{\bar{M}_{n}}$} & \multicolumn{3}{|c|}{ Tacticity $/ \%^{\mathrm{c}}$} & \multirow{2}{*}{$P_{m / r}^{\mathrm{d}}$} & \multirow{2}{*}{$P_{r / m}^{\mathrm{d}}$} \\
\hline & & & & $m m$ & $m r$ & $r r$ & & \\
\hline None & 100 & 7010 & 1.07 & 7.7 & 88.6 & 3.7 & 0.852 & 0.924 \\
\hline $\mathrm{CH}_{3}-\underset{0}{\mathrm{C}}-\mathrm{OC}_{2} \mathrm{H}_{5}$ & 68 & 5180 & 1.07 & 3.2 & 63.0 & 33.8 & 0.908 & 0.482 \\
\hline 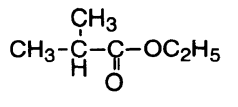 & 100 & 7700 & 1.06 & 5.9 & 77.2 & 16.9 & 0.867 & 0.695 \\
\hline${ }_{0} \mathrm{C}-\mathrm{OC}_{2} \mathrm{H}_{5}$ & 100 & 8240 & 1.06 & 6.5 & 89.2 & 4.3 & 0.873 & 0.912 \\
\hline $\mathrm{F}_{2} \mathrm{H}_{5}$ & 99 & 8170 & 1.12 & 7.5 & 88.9 & 3.6 & 0.856 & 0.925 \\
\hline $\begin{array}{c}\mathrm{CH}_{3} \\
\mathrm{CH}_{3}-\mathrm{C}-\mathrm{C}-\mathrm{OCH}_{3} \\
\mathrm{H}\end{array}$ & 100 & 7190 & 1.08 & 6.2 & 80.9 & 12.9 & 0.867 & 0.758 \\
\hline 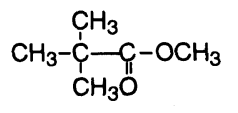 & 97 & 8540 & 1.06 & 6.6 & 89.0 & 4.4 & 0.871 & 0.910 \\
\hline$-\mathrm{OCH}_{3}$ & 100 & 8710 & 1.07 & 6.8 & 89.0 & 4.2 & 0.867 & 0.914 \\
\hline
\end{tabular}

a $t$-BuLi $0.2 \mathrm{mmol}, \mathrm{MeAl}(\mathrm{ODBP})_{2} 1.0 \mathrm{mmol}$, ester compound $1.0 \mathrm{mmol}$, EMA $10 \mathrm{mmol}$, toluene $10 \mathrm{ml}$. Ester compounds were added at $10 \mathrm{~min}$ after initiation. ${ }^{\mathrm{b}}$ Determined by GPC. ${ }^{\mathrm{c}}$ Determined by ${ }^{13} \mathrm{C}$ NMR. ${ }^{\mathrm{d}}$ The conditional probabilities in the first-order Markovian statistics.

improving heterotactic-selectivity.

\section{RESULTS AND DISCUSSION}

In Table I are summarized the results of polymerizations of MMA with $t$-BuLi/MeAl(ODBP $)_{2} /$ ester com-

Polym. J., Vol. 30, No. 9, 1998 pound $(1: 5: 5, \mathrm{~mol} / \mathrm{mol} / \mathrm{mol})$ in toluene at $-78^{\circ} \mathrm{C}$. In all the cases heterotactic polymers were obtained. Variation of the tacticity of the polymers obtained is relatively small $(m r=64.0-69.2 \%)$ irrespective of the bulkiness of the esters.

The polymerizations of EMA were also carried out 


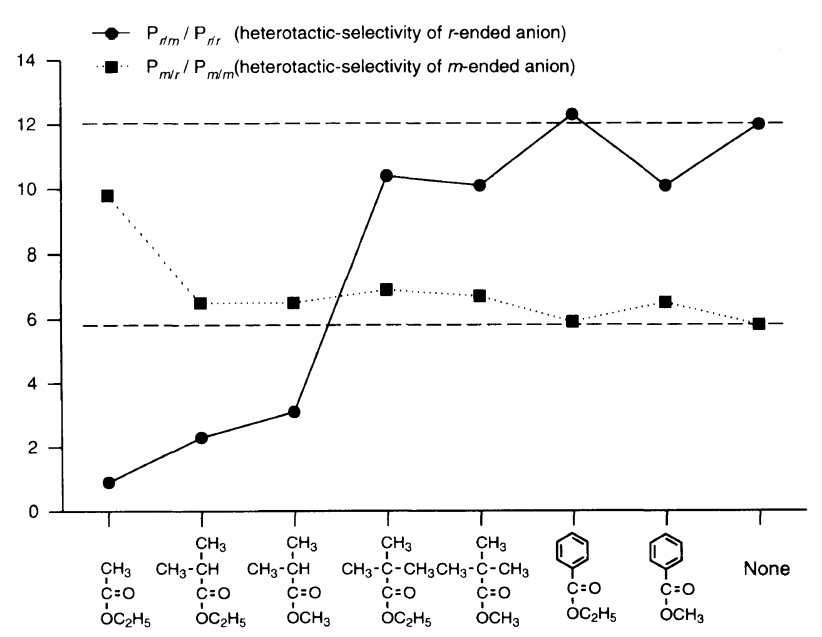

Figure 1. Heterotactic-selectivities of $m$-ended and $r$-ended anions in EMA polymerizations with $t$ - $\mathrm{BuLi} / \mathrm{MeAl}(\mathrm{ODBP})_{2}$ in the absence or presence of ester compounds.

under the same conditions. The results are summarized in Table II. Heterotactic polymers were obtained with narrow MWD. Stereoregularities of polymers obtained were affected significantly by the addition of ester compounds as compared with the cases of MMA, and $m r$ content varies from $63.0 \%$ (ethyl acetate) to $89.2 \%$ (ethyl pivalate).

Stereochemical processes of the heterotactic polymerization may be characterized by two parameters; the probability of $r$-addition by $m$-ended anion $\left(P_{m / r}\right)$ and that of $m$-addition by $r$-ended anion $\left(P_{r / m}\right)$ in first-order Markovian statistics. The ratios of the conditional probabilities, $P_{m / r} / P_{m / m}$ and $P_{r / m} / P_{r / r}$, can be regarded as parameters that represent heterotactic-selectivity of $m$ ended anions and that of $r$-ended anions, respectively. In Figure 1 are plotted the heterotactic-selectivities of $m$-ended and $r$-ended anions. The plots clearly demonstrate that the addition of the esters changes the heterotactic-selectivity of $r$-ended anions drastically but only slightly that of $m$-ended anions. The plots also indicate that the heterotactic-selectivity of $r$-ended anions depends on the bulkiness of the added esters; less bulky esters such as acetates and isobutyrates decrease the selectivity of $r$-ended anions while bulky esters such as pivalates and benzoates do not sacrifice the high selectivity of $r$-ended anions.

The remarkable difference between isobutyrates and pivalates is quite interesting. To examine this, coordination ability of ethyl isobutyrate and ethyl pivalate with $\mathrm{MeAl}(\mathrm{ODBP})_{2}$ was studied by ${ }^{13} \mathrm{C}$ NMR spectroscopy. Figure 2 shows ${ }^{13} \mathrm{C}$ NMR signals of carbonyl carbons of ethyl isobutyrate and ethyl pivalate in the presence or absence of an equimolar amount of $\mathrm{MeAl}(\mathrm{ODBP})_{2}$. In both cases, the carbonyl carbon signals showed downfield shift by the addition of $\mathrm{MeAl}(\mathrm{ODBP})_{2}$, indicating that both the ester compounds have the ability to form complexes with $\mathrm{MeAl}(\mathrm{ODBP})_{2}$. The results imply the possibility that these esters existing in the polymerization system may compete with EMA monomer in the coordination with $\mathrm{MeAl}(\mathrm{ODBP})_{2}$. Thus the NMR spectroscopic coordination experiments were also carried out for the mixtures of EMA and ester compounds. Figure 3 shows the ${ }^{13} \mathrm{C}$ NMR signals of the equimolar mixtures

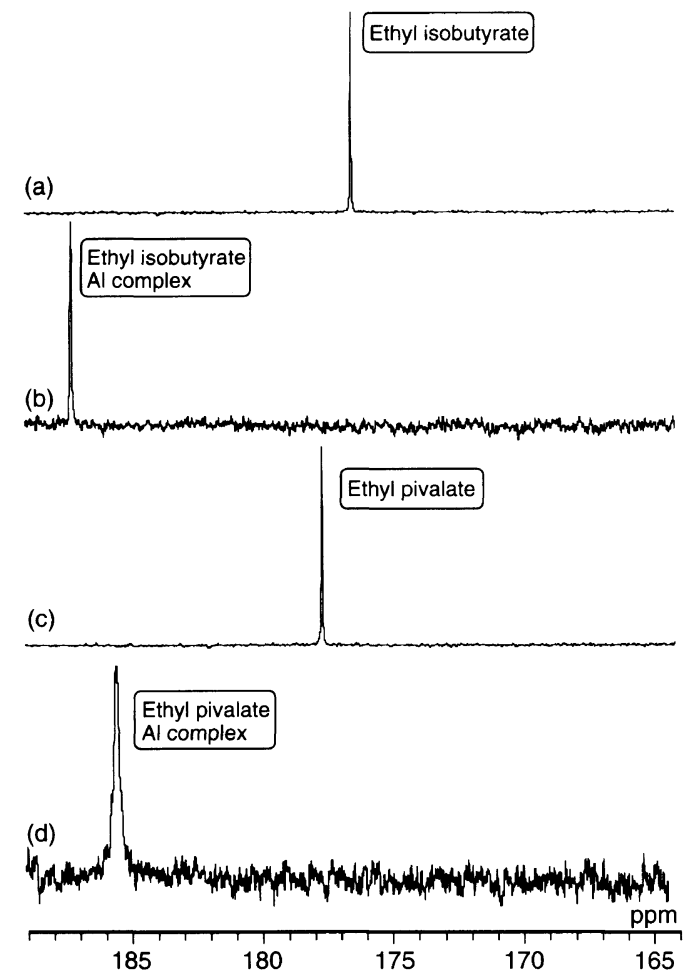

Figure 2. $67.8 \mathrm{MHz}{ }^{13} \mathrm{C}$ NMR spectra, measured in toluene- $d_{8}$ at $-78^{\circ} \mathrm{C}$, of carbonyl carbon of (a) ethyl isobutyrate, (b) ethyl pivalate, (c) ethyl isobutylate $/ \mathrm{MeAl}(\mathrm{ODBP})_{2}$, and (d) ethyl pivalate/MeAl$(\mathrm{ODBP})_{2}$

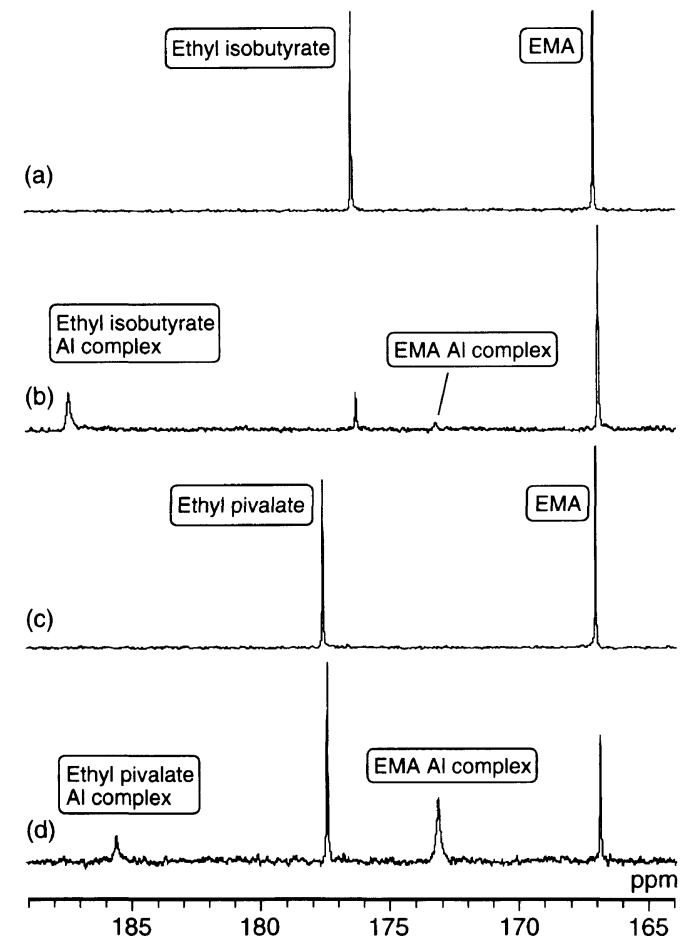

Figure 3. $67.8 \mathrm{MHz}{ }^{13} \mathrm{C}$ NMR spectra, measured in toluene- $d_{8}$ at $-78^{\circ} \mathrm{C}$, of carbonyl carbon of mixtures of (a) ethyl isobutyrate/EMA, (b) ethyl pivalate/EMA, (c) ethyl isobutylate/EMA/MeAl(ODBP $)_{2}$, and (d) ethyl pivalate/EMA/MeAl(ODBP $)_{2}$.

of EMA and the ester in the presence or absence of $\mathrm{MeAl}(\mathrm{ODBP})_{2}$. The ternary mixtures of EMA, ethyl isobutyrate or pivalate, and $\mathrm{MeAl}(\mathrm{ODBP})_{2}$ showed four carbonyl carbon signals, indicating that EMA and the 
ester compounds competitively coordinate to $\mathrm{MeAl}$ $(\mathrm{ODBP})_{2}$. In the case of ethyl isobutyrate (Figure 3(b)), the intensity of the signal at $173.1 \mathrm{ppm}$ due to the coordinated EMA is much weaker than that at $187.3 \mathrm{ppm}$ due to the coordinated isobutyrate. The results mean that $\mathrm{MeAl}(\mathrm{ODBP})_{2}$ mainly coordinates with ethyl isobutyrate rather than EMA. The selectivity of coordination was determined to be approximately EMA : ethyl isobutyrate $=2: 8 .{ }^{13}$ In the case of ethyl pivalate (Figure $3(\mathrm{~d})$ ), the intensity of the signal at $173.1 \mathrm{ppm}$ is a little stronger than that at $185.5 \mathrm{ppm}$ due to the coordinated pivalate, indicating that $\mathrm{MeAl}(\mathrm{ODBP})_{2}$ comparably coordinates with both ethyl pivalate and EMA; the selectivity of coordination was approximately EMA: ethyl pivalate $=6: 4 .{ }^{13}$ The latter result is consistent with the fact that triisobutylaluminum coordinates comparably to both methyl pivalate and MMA. ${ }^{14}$

The ratio of $\mathrm{MeAl}(\mathrm{ODBP})_{2}$ and $t$ - $\mathrm{BuLi}(\mathrm{Al} / \mathrm{Li})$ is also important to obtain the heterotactic polymers. When $\mathrm{Al} / \mathrm{Li}=1$, a syndiotactic polymer is obtained in low yield, while heterotactic polymers are obtained quantitatively at $\mathrm{Al} / \mathrm{Li} \geq 2 .{ }^{9}$ This result suggests that $\mathrm{MeAl}(\mathrm{ODBP})_{2}$ plays two significant roles in heterotactic polymerization; the stabilization of the propagating anions and the activation of the monomer. And the propagation reaction takes place selectively between the stabilized anion and the activated monomer (eq 3). To explain this peculiar

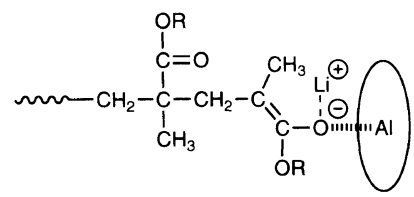

Stabilized anion

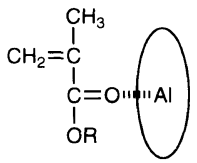

stereoregulation, we have postulated a mechanism where the presence or absence of $\mathrm{MeAl}(\mathrm{ODBP})_{2}$ at the penultimate ester group is the key; (1) there exist two types of propagating anions, one is coordinated by two MeAl$(\mathrm{ODBP})_{2}$ molecules at the chain end and the carbonyl group of the penultimate monomeric unit [A], and the other by one aluminum phenoxide at the chain end [B], and (2) $r$-ended anions, which should undergo $m$-addition, favor to be the former [A] and $m$-ended one, which should undergo $r$-addition, to be the latter $[\mathrm{B}] .^{15,16} \mathrm{Just}$ after monomer insertion, the newly formed anion may be type [A] and the anion [A] can be transformed to [B] by releasing the aluminum phenoxide having coordinated with the penultimate unit (Scheme 1).

The heterotactic-selectivity of $m$-ended anions in EMA polymerizations increased slightly by the addition of all the esters examined. The results are consistent with the above-mentioned assumption; that is, the addition of esters makes it easier for type [A] anion to be transformed to type [B] which favors $r$-addition. On the other hand, changes of the selectivity of $r$-ended anions are more complex. Bulky esters such as pivalate and benzoate do not diminish the high selectivity of $r$-ended anions, while less bulky ester, such as acetate and isobutyrate, decreased it significantly. These results mean that bulky esters form the complex selectively with the aluminum phenoxide which has coordinated with the penultimate

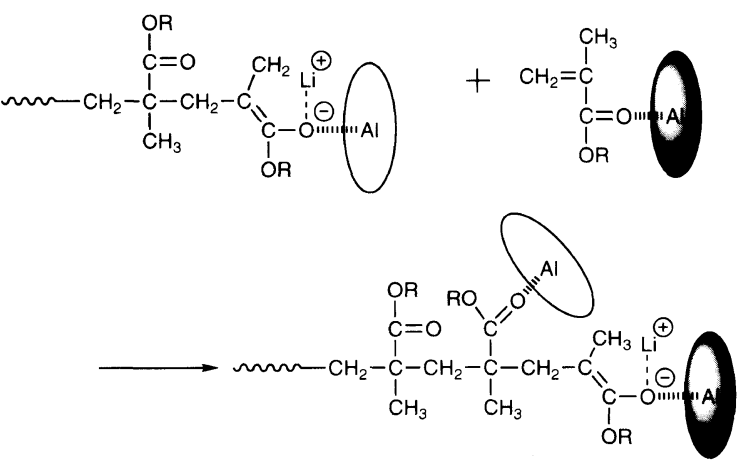

[A]

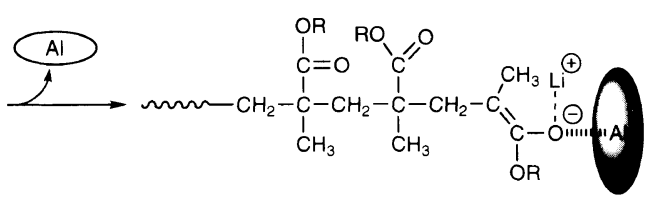

[B]

Scheme 1.

unit of $m$-ended anions rather than $r$-ended anion so that $r$-ended anion kept its high heterotactic-selectivity (Scheme 2). However, less bulky esters form the complex with that of not only $m$-ended anion but also $r$-ended anion so that $r$-ended anion decreased drastically its heterotactic-selectivity.

The heterotactic-selectivity of $r$-ended anions is higher than that of $m$-ended anions in EMA polymerizations, and vice versa in MMA polymerizations. However, the addition of less bulky esters such as acetate and isobutyrate into EMA polymerization systems made the heterotactic-selectivity of $r$-ended anion lower than that of $m$-ended anion as in MMA polymerizations. This indicates that not only these less bulky esters but also MMA itself prefer to form the complex with MeAl$(\mathrm{ODBP})_{2}$ coordinating with the penultimate unit (Scheme 3 ), and supports the fact that in MMA polymerization stereoselectivities of both anions were hardly influenced by the addition of ester compounds.

When ethyl acetate, the least bulky ester, was added, the polymer yield decreased in the polymerizations of MMA and EMA. This suggests that ethyl acetate forms the complex with $\mathrm{MeAl}(\mathrm{ODBP})_{2}$ more preferably than the monomers so that the amount of activated monomers decreases in these polymerization systems.

Lowering the polymerization temperature enhances heterotactic-selectivities of both $m$ - and $r$-ended anions, in particular, that of $r$-ended anions more evidently. ${ }^{11}$ As described above, the addition of the bulky esters enhances heterotactic-selectivity of $m$-ended anions, without loosing the high stereoselectivity of $r$-ended anion. Thus combining these two factors might further improve the heterotactic-selectivity. Table III summarizes the results of polymerization of EMA with $t$-BuLi/ $\mathrm{MeAl}(\mathrm{ODBP})_{2} /$ ester compounds at $-95^{\circ} \mathrm{C}$. The heterotactic-selectivity of $m$-ended anion increased slightly and that of $r$-ended anion was kept high enough, resulting in the increase of heterotacticity of the polymer obtained. However, the effect of the addition of the ester compounds in the polymerizations at $-95^{\circ} \mathrm{C}$ is less evident 


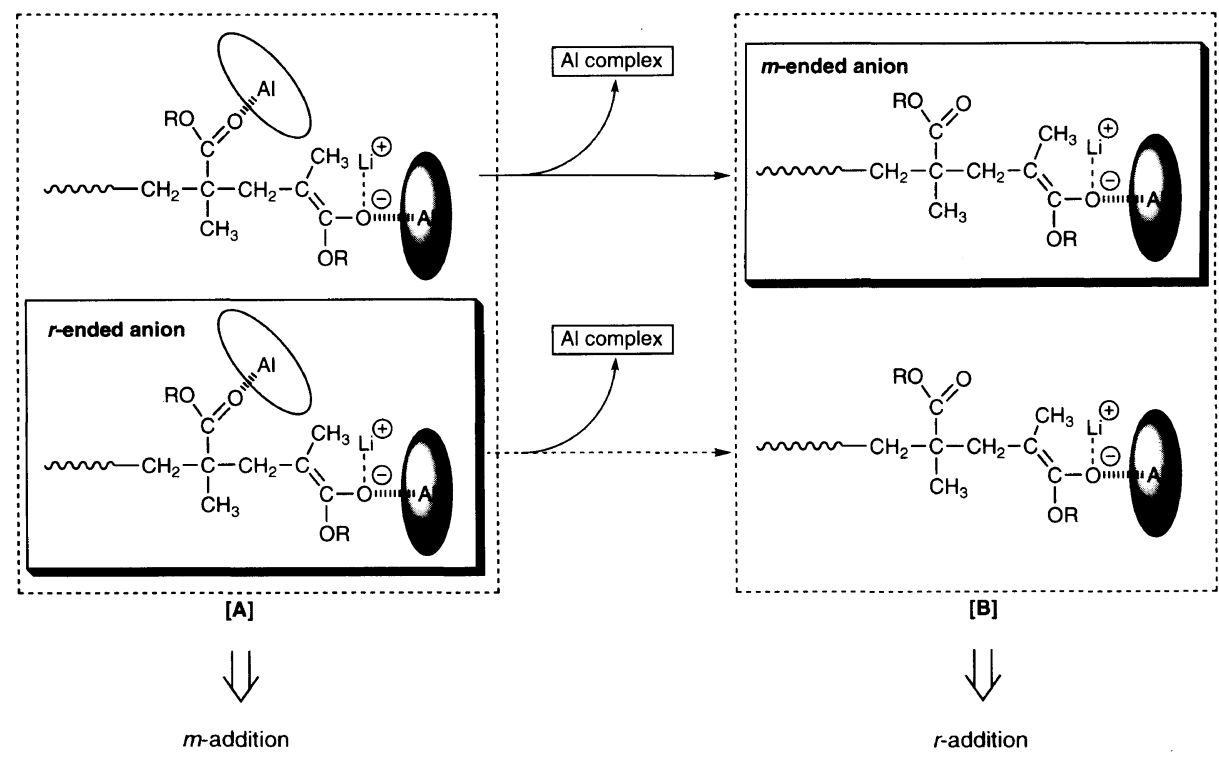

Scheme 2.

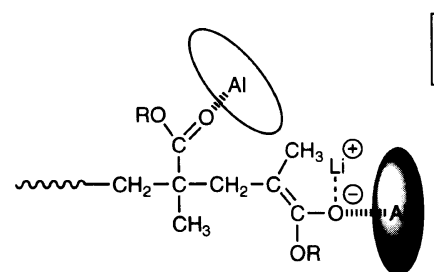

[A]

$\downarrow$

m-addition

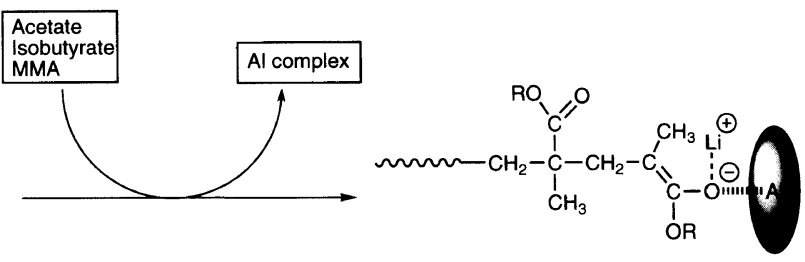

[B]

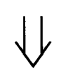

$r$-addition

Scheme 3.

Table III. Polymerization of EMA with $t$-BuLi/MeAl(ODBP) $)_{2} /$ ester compound in toluene at $-95^{\circ} \mathrm{C}$ for $48 \mathrm{~h}^{\mathrm{a}}$

\begin{tabular}{|c|c|c|c|c|c|c|c|c|c|c|}
\hline \multirow{2}{*}{ Ester } & \multirow{2}{*}{$\frac{\text { Yield }}{\%}$} & \multirow{2}{*}{$\bar{M}_{n}^{\mathrm{b}}$} & \multirow{2}{*}{$\frac{\bar{M}_{w}{ }^{\mathrm{b}}}{\bar{M}_{n}}$} & \multicolumn{3}{|c|}{ Tacticity $/ \%^{\mathrm{c}}$} & \multirow{2}{*}{$P_{m / r}^{\mathrm{d}}$} & \multirow{2}{*}{$P_{r / m}{ }^{\mathrm{d}}$} & \multicolumn{2}{|c|}{ Heterotactic-selectivity } \\
\hline & & & & $\mathrm{mm}$ & $m r$ & $r r$ & & & $\begin{array}{l}m \text {-Ended } \\
\text { anion }\end{array}$ & $\begin{array}{l}r \text {-Ended } \\
\text { anion }\end{array}$ \\
\hline None & 100 & 11100 & 1.07 & 7.0 & 92.0 & 1.0 & 0.868 & 0.979 & 6.6 & 46.0 \\
\hline $\begin{array}{c}\mathrm{CH}_{3}-\underset{\mathrm{C}}{\mathrm{C}}-\mathrm{H} \\
\stackrel{\mathrm{C}}{\mathrm{C}}-\mathrm{H}_{3} \mathrm{O} \\
\mathrm{C}\end{array}$ & 100 & 9430 & 1.08 & 6.8 & 92.2 & 1.0 & 0.871 & 0.979 & 6.8 & 46.1 \\
\hline$\Longrightarrow-\mathrm{C}-\mathrm{OC}_{2} \mathrm{H}_{5}$ & 99 & 10520 & 1.08 & 6.7 & 92.2 & 1.1 & 0.873 & 0.977 & 6.9 & 41.9 \\
\hline
\end{tabular}

${ }^{\mathrm{a}} t$-BuLi $0.2 \mathrm{mmol}, \mathrm{MeAl}(\mathrm{ODBP})_{2} 1.0 \mathrm{mmol}$, ester compound $1.0 \mathrm{mmol}$, EMA $10 \mathrm{mmol}$, toluene $10 \mathrm{ml}$. Ester compounds were added at $10 \mathrm{~min}$ after initiation. ${ }^{b}$ Determined by GPC. ${ }^{c}$ Determined by ${ }^{13} \mathrm{C}$ NMR. ${ }^{\mathrm{d}}$ The conditional probabilities in the first-order Markovian statistics. ${ }^{\text {e }}$ Calculated from $P_{m / r} / P_{m / m}$ and $P_{r / m} / P_{r / r}$.

as compared with the polymerization at $-78^{\circ} \mathrm{C}$. At $-95^{\circ} \mathrm{C}$, both anions form more stable structure so that the equimolar amount of ester compound with $\mathrm{MeAl}(\mathrm{ODBP})_{2}$ seems not enough to affect the stereoselectivity of the propagating species significantly. Then we investigated the use of a large amount of ethyl pivalate as a component of mixed solvent with toluene.

Table IV summarizes the results of polymerization of EMA in the mixtures of toluene and ethyl pivalate. When toluene and ethyl pivalate were used at $7 / 3$ (vol/vol) ratio, heterotactic poly(EMA) with $m r=94.3 \%$ was obtained. Figure 4 shows the carbonyl carbon NMR spectrum of the poly(EMA) together with that of the polymer with $m r=92.0 \%$ prepared in toluene. Integration curves are included to show a small but evident difference in tacticity. Both spectra showed strong peak due to rmrmrm heptad and small peaks, in particular, three weak peaks due to the defective sequences containing $\mathrm{mm}$ triad. In fact, the polymer with $m r=94.3 \%$ showed weaker peak intensity in the region due to $r m m r$ than the polymer prepared in toluene.

As the fraction of ethyl pivalate in the mixed solvent increased, the polymer yield decreased (Table IV). This suggests that $\mathrm{MeAl}(\mathrm{ODBP})_{2}$ competitively coordinates 
Heterotactic Polymerization of Methacrylates

Table IV. Polymerization of EMA with $t$-BuLi/MeAl(ODBP $)_{2}$ in toluene/ethyl pivalate at various ratio at $-95^{\circ} \mathrm{C}$ for $48 \mathrm{~h}^{\mathrm{a}}$

\begin{tabular}{|c|c|c|c|c|c|c|c|c|c|c|}
\hline \multirow{2}{*}{$\begin{array}{l}\text { Solvent } \\
\text { toluene: ethyl pivalate }\end{array}$} & \multirow{2}{*}{$\frac{\text { Yield }}{\%}$} & \multirow{2}{*}{$\bar{M}_{n}^{\mathrm{b}}$} & \multirow{2}{*}{$\frac{\bar{M}_{w}{ }^{\mathrm{b}}}{\bar{M}_{n}}$} & \multicolumn{3}{|c|}{ Tacticity $/ \%^{c}$} & \multirow{2}{*}{$P_{m / r}^{\mathrm{d}}$} & \multirow{2}{*}{$P_{r / m}^{\mathrm{d}}$} & \multicolumn{2}{|c|}{ Heterotactic-selectivity } \\
\hline & & & & $m m$ & $m r$ & $r r$ & & & $\begin{array}{l}m \text {-Ended } \\
\text { anion }\end{array}$ & $\begin{array}{l}r \text {-Ended } \\
\text { anion }\end{array}$ \\
\hline $10: 0$ & 100 & 11100 & 1.07 & 7.0 & 92.0 & 1.0 & 0.868 & 0.979 & 6.6 & 46.0 \\
\hline $9: 1$ & 81 & 7670 & 1.08 & 5.9 & 93.5 & 0.6 & 0.888 & 0.987 & 7.9 & 77.9 \\
\hline $7: 3$ & 51 & 9220 & 1.08 & 5.2 & 94.3 & 0.5 & 0.901 & 0.990 & 9.1 & 94.3 \\
\hline $5: 5$ & 27 & 4630 & 1.07 & 7.4 & 92.4 & 0.2 & 0.862 & 0.996 & 6.2 & 231.0 \\
\hline
\end{tabular}

${ }^{\text {a }} t$-BuLi $0.2 \mathrm{mmol}, \mathrm{MeAl}(\mathrm{ODBP})_{2} 1.0 \mathrm{mmol}$, EMA $10 \mathrm{mmol}$, solvent $10 \mathrm{ml}$. Polymerization was started by adding $t$-BuLi. ${ }^{\mathrm{b}}$ Determined by GPC. $\quad{ }^{\mathrm{c}}$ Determined by ${ }^{13} \mathrm{C}$ NMR. ${ }^{\mathrm{d}}$ The conditional probabilities in the first-order Markovian statistics. ${ }^{\mathrm{e}}$ Calculated from $P_{m / r} / P_{m / m}$ and $P_{r / m} / P_{r / r}$

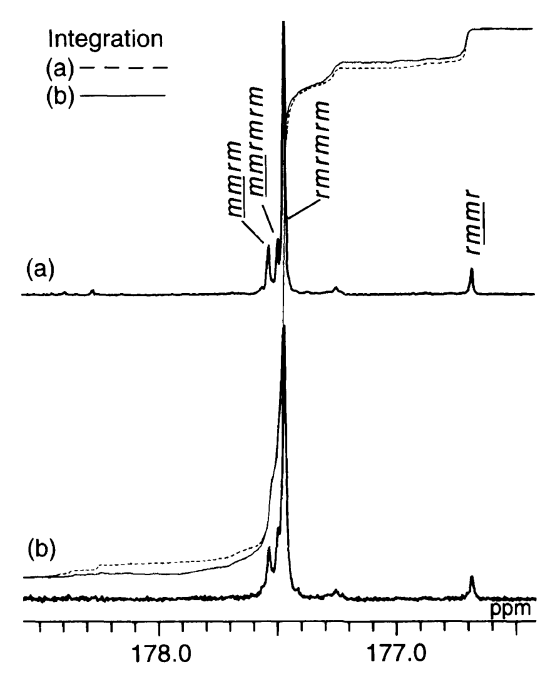

Figure 4. $125 \mathrm{MHz}{ }^{13} \mathrm{C}$ NMR spectra, measured in nitrobenzene- $d_{5}$ at $110^{\circ} \mathrm{C}$, of carbonyl carbon of heterotactic poly(EMA)s prepared with $t-\mathrm{BuLi} / \mathrm{MeAl}(\mathrm{ODBP})_{2}$ at $-95^{\circ} \mathrm{C}$ in toluene/ethyl pivalate $=($ a) $10 / 0$ and (b) $7 / 3$.

with EMA and ethyl pivalate so that the amount of activated EMA decreases. Although the added esters are expected, from the results shown in Table II, to decrease the heterotactic-selectivity of $r$-ended anions, the $r r$ content decreased smoothly as the fraction of ethyl pivalate increased. This result implies that the addition of a large amount of ethyl pivalate brings about not the effect in molecular level as mentioned above but the effect in bulk level, i.e., solvent effect. On the other hand, the $\mathrm{mm}$ content decreased with increasing the fraction of ethyl pivalate as expected, but the $\mathrm{mm}$ content increased again to $7.4 \%$ at the ratio of toluene and ethyl pivalate of $5 / 5$. The latter result reflects the decrease of $r$-selectivity of $m$-ended anions, which also implies the solvent effect by ethyl pivalate. If the propagating anions perform the stereoselection as in Scheme 2, these results suggest that the presence of a large amount of ethyl pivalate suppresses the removal of $\mathrm{MeAl}(\mathrm{ODBP})_{2}$ from the penultimate unit. The reason is not clear, but one possible explanation is that $\mathrm{MeAl}(\mathrm{ODBP})_{2}$ solvated with ethyl pivalate behaves as bulkier species due to the lyosphere and thus becomes more reluctant to leave from the penultimate unit of propagating anions. Since ethyl pivalate is not effective to remove $\mathrm{MeAl}(\mathrm{ODBP})_{2}$ from the penultimate unit of $r$-ended anion at $-95^{\circ} \mathrm{C}$ (the effect in molecular level) (see Table III), the increase in the fraction of ethyl pivalate seems to be reflected directly on the $m$-selectivity of $r$-ended anion (solvation effect). On the other hand, ethyl pivalate might remove $\mathrm{MeAl}(\mathrm{ODBP})_{2}$ from the penultimate unit of $m$-ended anion, where the molecular level effect is still operative. Thus the $r$-selectivity of $m$-ended anions increased smoothly as expected. However, as the fraction of ethyl pivalate increases further, the solvation effect becomes more important than the effect in molecular level. Consequently, $r$-selectivity of $m$-ended anions shows a maximum $(m r=94.3 \%)$ at the ratio of toluene and ethyl pivalate of $7 / 3$.

Recently, we found that allyl methacrylate gives heterotactic polymer with $m r=95.8 \%$ which is the highest heterotacticity among that of heterotactic polymers so far reported. ${ }^{17}$ Based on the findings about the solvent effect described in the present paper, the appropriate conditions for heterotactic polymerization of allyl methacrylate is now under examination to attain higher heterotacticity.

\section{EXPERIMENTAL}

\section{Materials}

MMA and EMA were purified by fractional distillation under reduced nitrogen pressure, dried over calcium dihydride and vacuum-distilled just before use. Toluene and heptane were purified in the usual manners, mixed with a small amount of $n$-BuLi, and distilled under high vacuum.

2,6-Di-t-butylphenol, obtained commercially, was fractionally distilled, dried over Molecular Sieves (MS) $4 \mathrm{~A}$, and used as a heptane solution.

$t$-BuLi in pentane (Aldrich Co., Ltd.) was used as a heptane solution. The concentration was determined by titration with butan-2-ol. ${ }^{18}$ Trimethylaluminum (Nippon Aluminum Alkyls) was used as a toluene solution.

Ester compounds were purified by fractional distillation under dry nitrogen or under reduced nitrogen pressure, dried over MS 4A, and used for polymerization reactions. Ethyl pivalate was dried over calcium dihydride and vacuum-distilled just before use.

\section{Preparation of $\mathrm{MeAl}(\mathrm{ODBP})_{2}{ }^{19}$}

A solution of 2,6-di-t-butylphenol in heptane (2 equiv.) was added slowly to a toluene solution of trimethylaluminum ( 1 equiv.) at $0^{\circ} \mathrm{C}$. The resulting mixture was stirred at room temperature for $20 \mathrm{~h}$. The solvent was removed under vacuum to leave a yellowish solid. The product was recrystallized three times by dissolving it in hot heptane and cooling to $-30^{\circ} \mathrm{C}$. After most of heptane was removed by a syringe and the residual heptane was 
evaporated under vacuum to dryness, the solid residue was dissolved in toluene and used for the polymerization reactions.

\section{Polymerization}

All the polymerization were carried out in a glass ampoule filled with dried nitrogen passed through MS $4 \mathrm{~A}$ cooled at $-78^{\circ} \mathrm{C}$. A heptane solution of $t$-BuLi was added to an aluminum phenoxide in toluene at polymerization temperature. The polymerization reaction was initiated by adding the monomer slowly to this mixture at polymerization temperature. Every ester compound was added into the polymerization mixture at $10 \mathrm{~min}$ after initiation. ${ }^{20,21}$ The last polymerizations summarized in Table IV were initiated by adding a heptane solution of $t$-BuLi to the mixture of monomer, ethyl pivalate, toluene, and $\mathrm{MeAl}(\mathrm{ODBP})_{2}$ to suppress the possible reaction of $t$-BuLi with ethyl pivalate prior to start of polymerization. ${ }^{20}$ The reaction was terminated by adding methanol containing $\mathrm{HCl}$ at the polymerization temperature. The reaction mixture was poured into a large amount of hexane to precipitate the polymeric product. In the case of MMA polymerization, the precipitate was collected by filtration, washed with hexane, dilute $\mathrm{HCl}$ and water, successively, and dried under vacuum. In the case of EMA polymerization, the polymeric product was precipitated by cooling the hexane to $-78^{\circ} \mathrm{C}$ and organic impurities such as monomer and 2,6-di- $t$-butylphenol derived from $\mathrm{MeAl}(\mathrm{ODBP})_{2}$ were removed by repeated decantation. The product was redissolved in acetone and the solution was poured into a large amount of water to reprecipitate the polymeric product. The precipitate was collected by filtration, washed with dilute $\mathrm{HCl}$ and water, successively, and dried under vacuum.

\section{Measurements}

Tacticities of PMMAs were determined from $\alpha$-methyl signals in ${ }^{1} \mathrm{H}$ NMR spectra measured in $\mathrm{CDCl}_{3}$ at $55^{\circ} \mathrm{C}$ on a JEOL JNM GX270 spectrometer operated at $270 \mathrm{MHz}$ or on a JEOL AL400 spectrometer operated at $400 \mathrm{MHz}$. Tacticities of poly(EMA)s and several PMMAs were determined from integration intensity of carbonyl carbon signals, measured in $\mathrm{CDCl}_{3}$ at $55^{\circ} \mathrm{C}$ or in nitrobenzene- $d_{5}$ at $110^{\circ} \mathrm{C}$ on a Varian Unity Inova 500 spectrometer operated at $125 \mathrm{MHz}$. However, the signals due to chain-end units were observed in the region of $r r$ triad, thus tacticities were calculated with removal of peak intensity of the resonances due to chain-end units. The standard deviations of tacticity were within $0.44 \%$ for five runs. Molecular weight and its distribution of the polymers were determined by gel permeation chromatography (GPC) using a JASCO 880-PU chromatograph equipped with two Shodex GPC columns [KF-806L $(30 \mathrm{~cm} \times 0.8 \mathrm{~cm}) \times 2]$ using tetrahydrofuran as an eluent at $40^{\circ} \mathrm{C}$. The GPC chromatogram was calibrated against standard PMMA samples.

\section{REFERENCES AND NOTES}

1. V. Busico, P. Corradini, L. De Martino, A. Proto, and E. Albizzati, Makromol. Chem., 187, 1115 (1986).

2. N. Kashiwa and J. Yoshitake, Makromol. Chem., Rapid Commun., 4, 41 (1983)

3. M. Kakugo, T. Miyatake, Y. Naito, and K. Mizunuma, Macromolecules, 21, 314 (1988).

4. S. Aoshima and T. Higashimura, Polym. Bull., 15, 417 (1986).

5. S. Aoshima and T. Higashimura, Macromolecules, 22, 1009 (1989).

6. T. Kitayama, T. Shinozaki, T. Sakamoto, M. Yamamoto, and K. Hatada, Makromol. Chem. Suppl., 15, 167 (1989).

7. H. Schlaad and A. H. E. Müller, Macromol. Symp., 107, 163 (1996).

8. H. Schlaad, B. Schmitt, A. H. E. Müller, S. Jüngling, and H. Weiss, Macromolecules, 31, 573 (1998).

9. T. Kitayama, Y. Zhang, and K. Hatada, Polym. J., 26, 868 (1994).

10. T. Kitayama, Y. Zhang, and K. Hatada, Polym. Bull., 32, 439 (1994).

11. T. Kitayama, T. Hirano, and K. Hatada, Polym. J., 28, 61 (1996).

12. T. Kitayama, T. Hirano, and K. Hatada, Polym. J., 28, 1110 (1996).

13. ${ }^{13} \mathrm{C}$ NMR spectra, obtained under complete-decoupling condition, usually do not allow quantitative analysis. Since the peak intensity ratios of EMA and esters, ethyl isobutyrate and ethyl pivalate, in the equimolar mixtures were $1: 0.9$ and $1: 1.2$, respectively. Though the values deviate $10-20 \%$ from the theoretical one, the results support that the difference of the isobutyrate and pivalate ( $c f$. Figures $3 \mathrm{~b}$ and $3 \mathrm{~d}$ ) is meaningful enough to discuss the selectivity of coordination from ${ }^{13} \mathrm{C}$ NMR spectra.

14. H. Schlaad, A.H. E. Müller, and H. Kolshorn, Macromol. Chem., Rapid Commun., 15, 517 (1994).

15. T. Kitayama, T. Hirano, and K. Hatada, Tetrahedron, 53, 15263 (1997).

16. T. Kitayama, T. Hirano, Y. Zhang, and K. Hatada, Macromol. Symp., 107, 297 (1996).

17. T. Hirano, H. Yamaguchi, T. Kitayama, and K. Hatada, Polym. J., 30, 767 (1998).

18. S. C. Watson and J. F. Eastham, J. Organomet. Chem., 9, 195 (1965).

19. A. P. Shreve, R. Mullhaupt, W. Fultz, J. Calabrese, W. Robbins, and S. D. Ittel, Organometallics, 7, 409 (1988).

20. When ester compounds, even bulky ester such as ethyl pivalate, were added before the addition of monomer, the initiator efficiency decreased to 0.38 from 0.87 for the opposite case described in the experimental section, probably because $t$-BuLi reacts with carbonyl group of esters.

21. T. Kitayama, T. Hirano, and K. Hatada, Polym. Prepr., Am. Chem. Soc., Div. Polym. Chem., 38, 504 (1997). 